\title{
A Fluorogenic Array Tag for Temporally Unlimited Single Molecule Tracking
}

\author{
${ }^{*}$ Rajarshi P. Ghosh ${ }^{1-4}$, *J. Matthew Franklin ${ }^{1-5}$, Will E. Draper ${ }^{1-4}$, Quanming Shi ${ }^{1-4}$, Jan T. \\ Liphardt $^{1-4}$ \\ ${ }^{1}$ Bioengineering, Stanford University, Stanford, CA 94305, USA \\ ${ }^{2}$ BioX Institute, Stanford University, Stanford, CA 94305, USA \\ ${ }^{3}$ ChEM-H, Stanford University, Stanford, CA 94305, USA \\ ${ }^{4}$ Cell Biology Division, Stanford Cancer Institute, Stanford, CA 94305, USA \\ ${ }^{5}$ Chemical Engineering, Stanford University, Stanford, CA 94305, USA \\ * Authors contributed equally.
}

Correspondence and requests for materials should be addressed to jan.liphardt@stanford.edu.

\begin{abstract}
Cellular processes take place over many timescales, prompting the development of precision measurement technologies that cover milliseconds to hours. Here we describe ArrayG, a bipartite fluorogenic system composed of a GFP-nanobody array and monomeric wtGFP binders. The free binders are initially dim but brighten 15 fold upon binding the array, suppressing background fluorescence. By balancing rates of intracellular binder production, photo-bleaching, and stochastic binder exchange on the array, we achieved temporally unlimited tracking of single molecules. Fast $(20-180 \mathrm{~Hz})$ tracking of ArrayG tagged kinesins and integrins, for thousands of frames, revealed repeated state-switching and molecular heterogeneity. Slow $(0.5 \mathrm{~Hz})$ tracking of single histones for as long as 1 hour showed fractal dynamics of chromatin. We also report ArrayD, a DHFR-nanobody-array tag for dual color imaging. The arrays are aggregation resistant and combine high brightness, background suppression, fluorescence replenishment, and extended choice of fluorophores, opening new avenues for seeing and tracking single molecules in living cells.
\end{abstract}

\section{Introduction}

Single molecule studies can reveal the operating principles of cellular machines ${ }^{1,2}$ but are difficult to perform in living cells. ${ }^{3,4}$ One problem is that fluorescent proteins (FPs) emit only a finite number of photons before bleaching, complicating data analysis and setting a fundamental limit on the description of biological systems. Consider a molecular machine traversing multiple microenvironments, while performing a series of operations and interacting with a succession of regulatory factors; a continuous $\mathbf{X}(\mathrm{t})$ track covering the entire process would give insight into reaction kinetics, the state space, transition probabilities, the noise structure, and the temporal order of molecular events.

One way to address limited observation times is to fuse multiple FPs to a $\operatorname{target}^{5}$ or to recruit FPs to arrays, as is done to track single RNA molecules and DNA loci. ${ }^{6-10}$ The RNA (or DNA) array approach can be extended to protein arrays, as demonstrated by the 'SunTag', an array of short epitopes that binds to single-chain GCN4 antibodies (scFv-GCN4). ${ }^{11}$ With such an 
approach, the key requirement is to maximize array occupancy while minimizing background signal by balancing relative expressions of the array and free binders. The SunTag reduces background fluorescence through spatial sequestration of free binders to the nucleus, presenting a challenge to nuclear protein tracking due to high backgrounds. ${ }^{12}$ The FP11-tag ${ }^{13}$ system reduces backgrounds using split-FPs, which have extremely slow maturation rates $\left(\sim\right.$ hours $\left.^{14}\right)$ and whose association is essentially irreversible. ${ }^{15-17}$ These characteristics allow FP11 to be used for bulk visualization of native proteins ${ }^{18}$ and advanced imaging such as complementation activated light microscopy ${ }^{16}$, but prolonged single molecule tracking (SMT) has not been not demonstrated.

We developed a class of genetically encoded array tags (Figure 1a-c) based on camelid nanobodies $^{19,20}$ and validated their intracellular performances. We demonstrate that low background fluorescence can be achieved using a fluorogenic array, where the fluorescence of the binder FP is minimal until it binds the array. One of the nanobody arrays, ArrayG, caused a 15-fold enhancement of wild-type GFP (wtGFP) fluorescence upon binding. A fully occupied 24x-GFP-nanobody array is thus $\sim 360$ fold brighter than a single wtGFP molecule. This approach yielded high signal to noise ratio (SNR) and allowed prolonged tracking of single molecules. To efficiently track single molecules in the nucleus, we designed a nuclear-specific derivative of ArrayG, termed ArrayGN. Under some imaging conditions, the continuous exchange of binders at the array with free mwtGFP allowed temporally unlimited tracking of single molecules without decay of the overall signal from the array for thousands of frames. A third array based on DHFR nanobody (ArrayD) allowed simultaneous dual color imaging

The primary consideration when using these array tags is their large size, which could restrict their applicability to large molecular complexes, or machines (e.g. kinesins) that have evolved to drag large payloads. However, both the moderately sized integrin $\beta 1(\sim 88 \mathrm{kDa})$ and histone H2B ( $\sim 14 \mathrm{kDa})$ tolerated the ArrayG/GN tag in a multitude of functional assays. For instance, $\beta 1$-ArrayG restored contractility to an integrin knockout cell line. The fluorogenic nature of the tag will potentially allow the use of shorter repeat arrays, where a tradeoff between array length and retention of native functionality will define the maximum achievable track duration.

\section{Results and Discussion}

Preliminary scan of possible array / binder pairs. We identified six high affinity protein interaction pairs in the literature (Supplementary Table 1). Five were nanobody-antigen pairs. The small size and monomeric nature of nanobodies facilitates generating tandem repeat arrays. ${ }^{19}$ The intracellular recruitment potential of each interaction pair was quantified using a nuclear compartmentalization assay, which was needed since in vivo affinities in cellular compartments can differ significantly from values determined in vitro (Supplementary Note 1, Supplementary Figure S1, Supplementary Table 1). We chose to further develop GBP1-GFP and NB113-DHFR, the two best performing interaction pairs. In particular, the GBP1-GFP binder pair provided a potentially attractive candidate for background fluorescence suppression due to GBP1's ability to enhance ${ }^{21}$ wtGFP fluorescence upon binding. 
Barrier 1: Aggregation. All multivalent recruitment strategies must contend with the possibility of aggregation. We encountered aggregation in our preliminary tests of the GBP1 nanobody array. In these early tests, we had fused a $24 x$ repeat of GPB1 (GBP1 $24 x)$ to KIF560, a (+) end directed kinesin-1 protein lacking the cargo-binding domain. ${ }^{5,22,23}$ Co-expression with eGFP resulted in numerous bright spots exhibiting complex dynamic behavior (Supplementary Movie S1. However, spot intensity analysis using calibrated confocal microscopy revealed higher-order aggregates of KIF560-GBB1 $1_{24 x}$, with a typical spot containing $\sim 1000$ eGFPs (Figure 1d, Supplementary Figure S2).

Addressing barrier 1: monomeric GFP. Aggregation was resolved by replacing eGFP, which has a weak dimerization propensity $\left(K_{d}=110 \mu \mathrm{M}\right)^{24}$, with the monomeric $A 206 K^{24}$ mutant ('mGFP'). This modification collapsed the previously broad distribution of intensities into two peaks with maxima corresponding to 21 and 38 GFPs (confocal microscopy) or 22 and 42 GFPs (HiLo-TIRFM) (Figure 1e-f). This likely represents a mixture of GBP1 ${ }_{24 x}-K I F 560$ homodimers and heterodimers of native kinesin 1 and GBP1 $1_{24 x}-\mathrm{KIF} 560$.

Barrier 2: Low throughput and variable background signal. The two requirements for attaining high signal-to-noise ratio (SNR) using a dynamic array strategy are high array

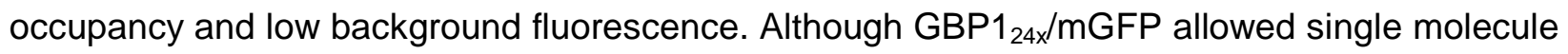
tracking in living cells, experimental throughput was initially low. Some cells were useable and provided good tracking data, but the vast majority of cells either had high backgrounds or poor signal.

Addressing barrier 2: Binding induced fluorescence enhancement for background suppression. To reconcile low background and high array occupancy, we explored a fluorogenic system in which where the free fluorophore is dark and only becomes fluorescent upon binding to the array. Single GBP1 nanobodies have been previously shown to enhance the fluorescence of wtGFP upon binding. ${ }^{21}$ We hypothesized that replacing mGFP with a monomeric A206K wtGFP (mwtGFP) would reduce background from free binders.

To compare mwtGFP and mGFP fluorescence, we expressed either mwtGFP or mGFP fused to mCherry via a P2A ${ }^{25}$ self-cleavable peptide (Figure 2a). Flow-cytometric analysis of the mCherry-normalized basal fluorescence showed $\sim 30$ fold less fluorescence for free mwtGFP compared to mGFP (Supplementary Figure S3). In contrast to FACS, HiLo-TIRFM imaging revealed an 18-fold weaker fluorescence for mwtGFP (5.5\% of the brightness of mGFP) (Figure 2b).

To measure the magnitude of the fluorogenic effect in the context of the GBP1 array, we co-expressed KIF560-GBP1 $1_{24 x}$ arrays in cells with either monomeric A206K wtGFP (mwtGFP) or mGFP. In fixed cells GBP1 $1_{24 x}$ was $\sim 75 \%$ as bright when occupied with mwtGFP than when occupied with mGFP (Figure 2c). Interestingly mwtGFP-bound $\mathrm{GBP}_{24 \mathrm{x}}$ brightened upon illumination even at relatively low laser powers $(<0.01 \mathrm{~kW} \mathrm{~cm})^{2}$ and photo-matured completely within $~ 200-300 \mathrm{~ms}$ (Figure 2c, inset). From these measurements we conclude that individual mwtGFPs undergo a net $\sim 15$-fold fluorescence enhancement upon binding the array (relative to mGFP, mwtGFP is $75 \%$ as bright when bound to GBP1 and $5.5 \%$ as bright when unbound).

To test whether the SNR of localized arrays was significantly improved by using 
mwtGFP, we compared cell lines expressing integrin $\beta 1-G B P 1_{16 x}$ and either mwtGFP or mGFP. We chose integrin $\beta 1$ over Kif560 to spatially confine the GBP1 scaffold to a single cellular compartment, facilitating quantitative comparison in live cells. Nearly every cell expressing mwtGFP exhibited arrays with high SNR (Supplementary Movie S2), whereas for mGFP single molecule detection was obscured in a large fraction of cells due to high background fluorescence (Figure 2d). Even when comparing mwtGFP cells to the subset of mGFP cells where single molecules could be detected, SNR increased significantly from 4.3 for mGFP to 5.8 for mwtGFP (Figure 2e). Moreover, when tracked under identical conditions, $\beta 1_{-G B P} 1_{16 \mathrm{X}}$ markedly increased track duration compared to $\beta 1$ - eGFP $2 x$ (Figure 2f) allowing tracking up to 105 seconds (2100 frames, Supplementary Movie S3). For ease of discussion, we refer to the $\mathrm{GBP}_{\mathrm{Nx}}+\mathrm{mwtGFP}$ as 'ArrayG $\mathrm{N}_{\mathrm{Nx}}$ ', where $\mathrm{N}$ represents the number of tandem repeats in the array.

Barrier 3: Photobleaching imposes a limit on trajectory length. Despite the extremely long track lengths of ArrayG, the tracks were still finite. However, a simple theoretical perspective on fluorescent binders that are stochastically exchanging on an array implies that in certain kinetic regimes temporally unlimited tracking is possible. Specifically, the average number of unbleached fluorophores $F$ bound to an array with $N$ binding sites will depend on the effective on rate $k_{\mathrm{on}}$, the off rate $k_{\mathrm{off}}$, and the bleach rate $k_{\mathrm{b}}$ (see Supplementary Note 2 for derivation).

$$
F=\frac{N}{1+\frac{k_{b}}{k_{o f f}}+\frac{k_{b}+k_{o f f}}{k_{o n}}}
$$

Using Eq. 1, we plotted the steady state array occupancy as a function of $k_{\text {off }}$ and $k_{\text {bleach }}$ assuming a large constant $k_{\text {on }}$. The plot shows a large parameter space in which $F$ can be driven close to $N$, given $k_{\text {off }}>>k_{\text {bleach }}$ and a non-limiting $k_{\text {on }}$ (Figure $3 a$ ). Experimentally, the photo-bleaching rate is primarily determined by exposure duration and frequency. By modulating exposure frequency one should be able to control bleach rate such that array fluorescence is indefinitely sustained.

Addressing barrier 3: Temporally unlimited trackability. Chromatin embedded fiducials are relatively immobile (compared to processive molecular motors and integrins), facilitating the evaluation of unlimited trackability. We chose histone H2B due to its extremely slow dissociation kinetics (multiple hours). ${ }^{26}$ For efficient nuclear import and integration of an H2B-ArrayG fusion, we generated 'ArrayGN', a modified version of ArrayG in which 3 copies of Snurportin-Importin $\beta$ binding domain $(\mathrm{IBB})^{27}$ were interspersed throughout the array (H2B-(Glycine-Serine repeat) $\left.{ }_{20 x}-\left(\mathrm{GBP}_{8 x}-\mathrm{IBB}\right)_{3 x}\right)$ (Figure 3b, Supplementary Movie 4).

We compared the photo-stability of H2B-ArrayGN in G1/S synchronized cells (Methods) imaged under continuous laser exposure versus pulsed exposure (50 ms exposure at $0.5 \mathrm{~Hz}$ ) (Figure 3b, c, Supplementary Movie 5) using identical laser power settings. Continuous illumination resulted in progressive decay of single H2B-ArrayGN intensities, whereas imaging under the pulsed exposure format showed no signs of intensity decay (Figure 3c, Supplementary Movie 5). Furthermore, when imaged after formaldehyde fixation of cells, spot intensity decayed similarly for both continuous and pulsed exposure (Figure 3c), demonstrating 
that stochastic turnover on the array was the fundamental mechanism of signal replenishment. Finally, we directly observed fluorescence recovery after photo-bleaching in both ensemble $\left(\mathrm{t}_{1 / 2}\right.$ $=27 \pm 9 \mathrm{~min}$ ) and single-molecule modes in cells stably expressing $\mathrm{H} 2 \mathrm{~B}-$ ArrayGN $_{24 \mathrm{X}}+$ mwtGFP $\left(t_{1 / 2}=23 \pm 4 \mathrm{~min}\right)$ (Figure 3d, Supplementary Figure S4, Methods), demonstrating that a fully bleached array will gradually recover its fluorescence due to stochastic turnover.

Intracellular performance tests and tag validation. Having developed a tag (Array $\left.\mathrm{G}_{24 \mathrm{x}}\right) 360$ fold brighter than wtGFP with three defining features, (1) aggregation resistance, (2) background suppression through fluorogenicity, and (3) indefinite trackability, we tested its performance in living cells.

Test 1: Multiscale dynamics of single chromatin bound histones in the living nucleus. We measured the dynamics of single $\mathrm{H} 2 \mathrm{~B}-$ Array $_{\mathrm{GN}} \mathrm{N}_{24}$ labeled nucleosomes in G1/S synchronized cells. The ArrayGN $\mathrm{N}_{24 \mathrm{x}}$ tags with their low backgrounds allowed sustained tracking of $\mathrm{H} 2 \mathrm{Bs}$ and yielded a large SMT dataset. For example, one cell with $194 \mathrm{H}_{2} \mathrm{~B}-\mathrm{ArrayGN}_{24 \mathrm{x}}$ molecules provided 147,000 seconds of precision $2 \mathrm{D}$ tracking data $(0.5 \mathrm{~Hz}$ imaging, mean track length $\sim 760$ seconds, maximum track length 4200 seconds). At the lower imaging rate $(0.5 \mathrm{~Hz})$, track lengths were no longer limited by loss of signal due to photobleaching; a track typically ended when a molecule moved out of the HiLo imaging volume. $X Y(t)$ data for three histones are shown in Figure 3e. The single histone trajectories had rich dynamics; for example, the nanometer vs. hour plot (bottom of Figure 3e) shows the displacements of one histone's position over more than 1 hour, encompassing a $4 x$ range of frame-to-frame displacements (Figure 3e, bottom).

While the technology should facilitate the detailed study of specific gene loci and chromatin dynamics (such as looping and TADs formation), our focus here are the baseline characteristics of living chromatin, especially its spatial and temporal geometry. Sequencing methods such as $\mathrm{Hi}-\mathrm{C}$ suggest that chromatin is spatially self-similar at length scales from $\sim 100$ kilobases to several megabases. ${ }^{28,}{ }^{29}$ Separately, there is evidence that chromatin is also temporally fractal; optical tracking of single loci in prokaryotes and eukaryotes suggests a Hurst parameter $H \sim 0.25$ (corresponding to an exponent $\alpha=2 H \sim 0.5$ ). ${ }^{30-33} \mathrm{H} 2 \mathrm{~B}$-ArrayGN imaged at $20 \mathrm{~Hz}$ and $0.5 \mathrm{~Hz}$ were fit to fractional Brownian motion (fBm) using a maximum likelihood criterion. The $0.5 \mathrm{~Hz}$ tracks yielded an exponent $\alpha=0.42 \pm 0.14$ (95\% confidence interval [0.41, $0.43]$ ) while the $20 \mathrm{~Hz}$ tracks yielded $\alpha=0.43 \pm 0.16$ (95\% confidence interval [0.42, 0.45], Figure 3f, Methods). Peripheral histones (nearer the nuclear envelope) were more confined than 'interior' histones (Supplementary Figure S5). The a and apparent diffusion coefficient estimates obtained from the chromatin-integrated ArrayGN-histones are in excellent agreement with the values obtained from short tracks of single GFP-tagged histones by Shinkai and coworkers. $^{34}$

The similarity of the a values for different systems (single GFP-H2B vs. H2B-ArrayGN at $20 \mathrm{~Hz}$ and $0.5 \mathrm{~Hz}$ ) suggests that the labeled histones are reporting the underlying motions of relatively large regions of the chromatin, whose molecular weight far exceeds that of any single protein (or protein complex). It is notable that recent estimates of $\alpha$ in eukaryotes tend to be closer to 0.4 than 0.5 , but these differences are well within the variation of \pm 0.1 seen across cell lines. $^{35}$ The fact that the short $(20 \mathrm{~Hz})$ trajectories, covering seconds to minutes, and the hour- 
timescale $(0.5 \mathrm{~Hz})$ trajectories give nearly identical a values strongly supports the notion that chromatin has a fractal temporal structure.

Test 2: Molecular motors/Kinesins. A second major application area for live cell SMT is the large field of motor mechano-chemistry and regulation. In contrast to the tethered nature of $\mathrm{H} 2 \mathrm{~B}$ dynamics, motor proteins exhibit relatively fast directional motion. To inspect the intricate details underlying the fast, directionally-persistent motion of motor proteins, we tracked KIF560Array $_{24 x}$ using HiLo-TIRFM at $20 \mathrm{~Hz}$. This yielded hundreds of directionally processive trajectories per cell, spanning up to several $\mu \mathrm{m}$ in length (Figure 4a, Supplementary Movie S6). Live staining of cells with the fluorogenic SiR-tubulin dye allowed us to track single molecules of kinesin on microtubule track for extended lengths of time at higher imaging rates $(80 \mathrm{~Hz})$ (Figure 4b, Supplementary Movie S7). The speed of KIF560-ArrayG ${ }_{24 x}$ motors was sharply distributed about a mean of $1.48 \mu \mathrm{m} / \mathrm{s}$ (Figure $4 \mathrm{c}-\mathrm{d}$ ). The average speeds and runlengths also matched existing live cell measurements. ${ }^{5,11}$

To extend the scope of array-based tags for simultaneous multi-color imaging, we developed an orthogonal array tag based on the nb113 DHFR-nanobody ${ }^{36}+E$. Coli DHFR arraybinder pair, termed ArrayD. One added advantage of ArrayD is that it can be used to recruit any FP as a fusion to DHFR. Despite a lower reported affinity ${ }^{36}$ of the nb113+DHFR pair compared to the GBP1+GFP pair, Array $D_{24 x}$ allowed efficient tracking of single kinesins when coexpressed with DHFR-mGFP (Supplementary Movie S8) or DHFR-mCherry (Supplementary Movie S9). The corresponding average speeds and track lengths were similar $(1.51 \mu \mathrm{m} / \mathrm{s}$ and $1.61 \mu \mathrm{m} / \mathrm{s}$ average speed, $0.94 \mu \mathrm{m}$ and $1.10 \mu \mathrm{m}$ average run length, for mGFP-DHFR and mCherry-DHFR, respectively) (Supplementary Figure S6). Furthermore, by introducing ArrayG $_{24 x}$ (with mWTGFP) and ArrayD ${ }_{24 x}$ (with DHFR-mCherry) in the same cell, we were able to simultaneously track two populations of KIF560 (Supplementary Movie S10). These gave indistinguishable average speeds $(1.54 \mu \mathrm{m} / \mathrm{s}$ and $1.56 \mu \mathrm{m} / \mathrm{s}$ for mGFP and mCherry, respectively) showing internally consistent results and demonstrating that these arrays are suitable for simultaneous multicolor imaging (Figure $\mathbf{4 e - g}$ ).

Highly resolved temporal dynamics of kinesin. The photo-stability and brightness of ArrayG also made it possible to capture high-frequency features of kinesin dynamics. Using a 25-fold higher laser illumination $\left(0.2 \mathrm{~kW} / \mathrm{cm}^{2}\right)$, we were able to track KIF560 at $180 \mathrm{~Hz}$, revealing up to 4 cycles of binding, directed motion, unbinding, and free diffusion in single trajectories (Figure $\mathbf{4 h}$, Supplementary Movie S11). During the periods of directed motion, the speed varied from less than $1 \mu \mathrm{m} / \mathrm{s}$ to nearly $3 \mu \mathrm{m} / \mathrm{s}$, with an average speed around $1.5 \mu \mathrm{m} / \mathrm{s}$ (Figure 4i). To our knowledge, such short-lived speed transients have not been previously reported using genetically-encoded tags. Precision, high-frequency tracking using ArrayG may help answer questions regarding adaptive changes in behavior of kinesin moving on a dynamic microtubule landscape ${ }^{37}$, particularly in living systems only accessible through the use of genetically encoded tags.

Test 3: Long-term imaging of integrin $\beta 1$ using ArrayG reveals novel mobility states and context-dependent state transition-rates. A third major application area of single molecule tracking is the study of transmembrane complexes. Previous single molecule studies have 
shown that transmembrane proteins exhibit multiple states of motion, corresponding to different functional states. ${ }^{38-40} \mathrm{~A}$ common limitation in these studies is trajectory duration, preventing a proper/thorough quantification of transient state-switching dynamics. To explore the utility of ArrayG for tracking transmembrane proteins, we fused it to integrin $\beta 1$. Previous short timescale SPT-PALM experiments have suggested that integrins transition between diffusive inactive conformations and immobile active conformations. ${ }^{40}$ We used four separate criteria to evaluate the functionality of $\beta 1$-ArrayG: enrichment at focal adhesions, functional complementation in integrin $\beta 1$-deficient cell lines, rate of diffusion, and response to $\mathrm{Mn}^{2+}$. We generated stable cell lines expressing a more compact derivative of ArrayG with 16 repeats (Array $\mathrm{G}_{16 \mathrm{x}}$ ) fused to integrin $\beta 1$ (Supplementary Note 3 ). Through trans-complementation of cells lacking native integrin $\beta 1$ with $\beta 1$-Array $G_{16 x}$ or $\beta 1-P 2 A-e G F P$ fusions we verified that $\beta 1-$ ArrayG $_{16 x}$ enriched at focal adhesions (Figure 5a) and restored wild type levels of cell/substrate engagement and spreading to a spreading-deficient integrin knockout line (Figure 5b-c, Supplementary Figure S7, Supplementary Note 3).

Quantification of diffusivity revealed enhanced immobilization of $\beta 1-$ Array $_{16 x}$ molecules inside adhesions marked by vinculin-mCherry (Figure 5d-e, Supplementary Figure S8). We observed the lateral diffusion of $\beta 1-$ Array $_{16 x}$ across adhesion boundaries and subsequent immobilization inside the adhesion (Figure 5e, Supplementary Movie S12), supporting the proposed 'archipelago' model of focal adhesion architecture. ${ }^{41}$

To assess the impact of the ArrayG tag on lateral diffusion within the membrane, we compared the dynamics of $\beta 1-$ ArrayG $_{16 x}$ to $\beta 1-\mathrm{eGFP}_{2 x}$ in $\mathrm{pKO}-\alpha \mathrm{V} \beta 1$ cells $^{42}$ that express endogenous integrin $\beta 1$. The diffusion coefficients had a bimodal distribution representing a freely diffusing and an immobilized population, in accordance with previous measurements ${ }^{40}$ (Figure 5f, Supplementary Figure S9). Notably mobile $\beta 1$-ArrayG ${ }_{16 x}$ molecules had a $1.9 x$ smaller diffusion coefficient compared to $\beta 1$-eGFP $2 x$. Considering the distribution of diffusivity for integrin $\beta 1$ spans over $\sim 5$ orders of magnitude, a $1.9 x$ reduction may not be functionally relevant, as demonstrated by the ability of $\beta 1-$ Array $_{16 x}$ to restore spreading to wild-type levels in a $\beta 1$ knockout cell line. Moreover, the shape and peaks of the two diffusivity distributions were nearly identical (Figure 5f).

We also checked whether $\beta 1-$ Array $_{16 x}$ could respond effectively to an external stimulus, by measuring response to the addition of $\mathrm{Mn}^{2+}$, a potent activator of integrins that enhances integrin immobilization. ${ }^{40}$ Stimulation with $2 \mathrm{mM} \mathrm{Mn}^{2+}$ for 1 hour immobilized both $\beta 1-\mathrm{eGFP}_{2 x}$ and $\beta 1-$ Array $_{16 x}$ to a similar extent (Figure $\mathbf{5 g}$, Supplementary Figure S9). In summary, Array $\mathrm{G}_{16 \mathrm{x}}$ fusion retained the biological functions of integrin $\beta 1$ in our controls.

For robust detection of states and accurate measurement of state dwell times and transition probabilities, single molecule trajectories need to be longer than the mean state lifetimes. ${ }^{43} 20 \mathrm{~Hz}$ imaging of $\beta 1-$ Array $_{16 x}$ provided an ideal balance between trajectory duration and temporal resolution. At this frequency, although photo-bleaching ultimately limited trajectory length, $\beta 1$-Array $\mathrm{G}_{16 \times}$ significantly increased track duration and throughput compared to previous integrin tracking reports ${ }^{40,44}$.

Starting with the a priori assumption that integrin $\beta 1$ dwells in three different diffusive states (diffusive, confined, and immobilized) ${ }^{40}$, we segmented trajectories using a variational Bayes treatment of hidden Markov models for single molecule trajectories (vbSPT). ${ }^{43}$ However, a four-state model improved the model evaluation score (Supplementary Note 4), revealing 
four distinct diffusion states (Figure 6a-b). The 4-state model suggested that integrin $\beta 1$ frequently visits a short-lived binding state, and may subsequently transition into a more confined and longer-lived super-bound state (Figure 6c, Supplementary Figure S10, Supplementary Note 4). Such detailed state dynamics have not been previously measured due to the lack of tools for high-throughput acquisition of long-lived trajectories. The multiple binding states are consistent with the multiple conformational states of integrin heterodimers ${ }^{45}$, and the multi-modal binding lifetimes of integrins under tension. ${ }^{46}$

To test whether the integrin $\beta 1$ state-landscape depended on local integrin density ${ }^{47,48}$ we analyzed trajectories of sparsely expressed $\beta 1$-Array $G_{16 x}$ in $\beta 1$-null cells which display impaired contractility $\left(\mathrm{pKOaV} \beta 1^{--/}\right)$. In contrast to cells expressing native $\beta 1$, bound state occupancy in $\beta 1$-null cells was significantly reduced, with more frequent transitions between the fast and slow diffusive states (Figure $6 \mathbf{d}$ ). These results demonstrate that $\beta 1-$ ArrayG $_{16 x}$ dynamics are sensitive to the biological context.

Although vbSPT analysis maybe performed on a large number of short trajectories ${ }^{43}$, the accuracy of the model improved when track lengths were larger than the state lifetimes (Supplementary note 4). We found that the various states of integrin mobility had average lifetimes on order of 1 second (ranging from 0.7 to 4.6 seconds), with the super-binder state lasting up to 49 seconds in one instance. This range of lifetimes underscores the importance of long trajectories for distinguishing between states with similar diffusion coefficients, but different lifetimes.

\section{Outlook}

Despite multiple fluorophore recruitment techniques (including SunTag ${ }^{11}$ and SpaghettiMonster ${ }^{49}$ ), the field currently lacks optical tagging systems with demonstrably long duration SMT capabilities. The ArrayG system utilizes a nanobody-fluorophore combination that is orthogonal to most eukaryotic complexes. The targets we studied (kinesins, integrins, histones) tolerated the array fusions and we did not see detriments to cell viability, cell division, or intracellular dynamics of the labeled targets. Despite the success with kinesins, integrins, and histones, any new target labeled with the Array tags should be critically evaluated for possible functional impairment.

By exploiting slow exchange of fluorophores at the array, we have found that single molecules of $\mathrm{H} 2 \mathrm{~B}-$ Array $\mathrm{GN}_{24 \mathrm{x}}$ can be optically tracked for very long times, limited only by biological factors such as movement of molecules out of the imaging volume. Note that the traditionally assumed tradeoff between imaging duration and localization precision can be circumvented, via stochastic turnover of FPs on an array, as demonstrated by ArrayG. As shown with the high frequency imaging of kinesins and integrins, the Array tags can also be used to track state transitions at high temporal resolution. The ability to track single biomolecules for temporally unlimited durations may prove useful for addressing questions in assembly, targeting, motility, protein turnover, and cell division. Finally, the fluorogenic ArrayG tag, might allow prolonged SMT in deep tissues where background fluorescence is particularly problematic. 


\section{Materials and Methods}

Array plasmid generation. All mammalian codon optimized Array monomeric unit-DNA were purchased from IDT (integrated DNA technologies) as genes in pUCIDT-Kan plasmid backbone or gBlock gene fragments. All monomeric units had the following structure: (Sall)-6bp spacerScaffold unit-flexible Linker-(Xhol)-6bp spacer-(BamHI). Using the surrounding Sall-Xhol-BamHI combination, we put the monomer unit through several rounds of repeat building, in which we doubled the number of direct repeats with each cloning cycle, as originally shown by Robinett et al. ${ }^{8}$ After repeat building, the monomer units were moved into a modified Clontech PEGFP-N1 backbone with a Tet-On 3G Tetracycline inducible promoter in place of the CMV promoter (Tet3G backbone). All repeat-containing plasmids were transformed and maintained in MAX Efficiency Stbl2 competent cells (Thermo Fisher Scientific) grown at $30{ }^{\circ} \mathrm{C}$, as long repeats were unstable in other cell types. For generating double stable cell lines, binder-FPs were inserted into the MCS of PiggyBac Cumate switch system (PBQM812A-1, SBI). For the array plasmids, PBQM812A-1 was modified to replace the cumate operator sequence (CuO) with a Tet response element, the EF1-CymR repressor-T2A-Puro cassette was replaced with a custom made EF1-Tet-Activator-T2A-Hygro cassette and the existing MCS was replaced with a custom MCS, such that Arrays could easily be moved from Tet3G backbone plasmids. Cells efficiently transposed for the array and the binder were puromycin and hygromycin positive respectively and were inducible with doxycycline (array) cumate (binder). Doxycycline control was leaky enough to produce arrays in amounts that were ideal for single molecule imaging, when cells were uninduced.

Integrin $\beta 1$, kinesin and $\mathrm{H} 2 \mathrm{~B}$ source plasmids were purchased from addgene (Addgene numbers 55064, 15284 and 20972), Vinculin-mCherry was a gift from Johan De Rooij. KIF560 (amino acids 1-560) was generated from full length kinesin. All plasmid sequences are available from NCBI GenBank with the following accession numbers: To be provided by GenBank.

Cell Culture. HeLa Tet-On® $3 G$ Cell Line (\#631183) and U2-OS Tet-On® Cell Line (\#631143) were purchased from Clontech. MEF-pKO lines (Shiller et al. ${ }^{42}$ ) were a gift from Alexander Dunn (Dept. of Chemical Engineering, Stanford University). All mammalian cells were cultured on tissue culture plastic in standard tissue culture incubators held at $37^{\circ} \mathrm{C}$ with $5.0 \% \mathrm{CO}_{2}$ using DMEM supplemented with $20 \%$ fetal bovine serum and penicillin-streptomycin antibiotics. Before plating $\mathrm{pKOaV}$ and $\mathrm{pKOaV} \beta 1$ mouse embryonic fibroblast cell lines, plastic cultureware was incubated with $5 \mu \mathrm{g} / \mathrm{mL}$ fibronectin for at least one hour and then washed with PBS before adding culture media and cells.

U2OS cells co-expressing H2B-ArrayGN and mWTGFP were synchronized to G1/S boundary using a double thymidine block procedure. $\sim 30 \%$ confluent cells were blocked with $2.5 \mathrm{mM}$ Thymidine for $\sim 20$ hours followed by a release step lasting 10 hours, followed by a second Thymidine block. Cells were treated with cumate during the 10-hour release step to induce expression of mWTGFP. For imaging cells in prophase, cells were released from the second thymidine block after a period of $\sim 16$ hours, incubated for 2 hours in fresh medium,

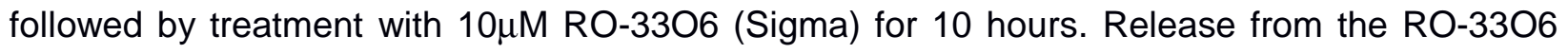
block allowed cells to synchronously enter mitosis. 
Transfections. All transfections were performed using the Neon Transfection system (Thermo Fisher Scientific). HeLa cells were co-transfected with 0.1-0.2 $\mu \mathrm{g}$ of binder plasmids and 4-8 $\mu \mathrm{g}$ of ArrayG or ArrayD plasmids. We generally used two electroporation pulses (pulse voltage $1,005 \mathrm{~V}$, pulse duration $35 \mathrm{~ms}$ ). pKO- $\alpha \mathrm{V} \beta 1$ as well as pKO- $\alpha \mathrm{V} \beta 1 \%$ cells were transfected using the following pulse program: 1 pulse, $1,350 \mathrm{~V}$ pulse voltage, $30 \mathrm{~ms}$ pulse width. U2OS cells were transfected using 2 pulses of $1,050 \mathrm{~V}$ and $30 \mathrm{~ms}$ pulse width. Upon transfection, cells were seeded on Mattek dishes coated with fibronectin (Sigma Aldrich) in complete DMEM media without Pen/Strep and with teteracycline free FBS (Thermo Fisher Scientific).

Cell Line generation. HeLa, U2OS and pKO-aV/aVß1 cell lines were generated by transfecting cells with the relevant PiggyBAC plasmids and a plasmid expressing transposase following manufacturer's protocol and then selecting using appropriate antibiotics. For single stable cell line puromycin was the antibiotic of choice where as for double stable cell lines a combination of puromycin and hygromycin was used.

FACS Analysis. Hela cells stably expressing mwtGFP-P2A-mCherry or mGFP-P2A-mCherry were generated as described above. The cells were analyzed with flow cytometry (BD LSR II) in GFP and mCherry channel along with blank control cells. Only singlets were selected and further analyzed. The ratio of GFP to mCherry per-cell were calculated and displayed after subtracting the background signal from control cells.

Single GFP quantification for occupancy estimation method 1: confocal calibration. We performed a multi-step calibration of a Zeiss LSM 700 laser scanning confocal so that we could estimate the number of GFPs within each fluorescent spot. First, we measured the bulk fluorescence of an eGFP solution over a wide variety of microscope settings, varying the PMT gain from 450 to 750 volts, confocal pinhole from 41 to $100 \mu \mathrm{m}$, and the laser from $1 \%$ to $5 \%$ power. We fit all the measurements to an arbitrary three-parameter 'bulk' model of the form Counts $=A \times$ laser $\times($ pinhole $-B) \times$ gain $^{C}$, allowing us to estimate the solution's fluorescence at arbitrary instrument settings (Supplementary Figure S2).

We measured the bulk per-molar fluorescence of purified eGFP (CellBioLabs), and 0.04 $\mu \mathrm{m}$ green-fluorescent bead standards ( $5 \% \mathrm{w} / \mathrm{v}$ FluoSpheres, Molecular Probes) by measuring fluorescence at several fluorophore solution concentrations, and several different microscope settings for redundancy (Supplementary Figure S2f, g). To measure bead molarity, which is simply particles per unit volume, beads were immobilized by forming a $5 \%$ poly-acrylamide gel within a hemacytometer (Incyto) so they could be counted within a known volume (Supplementary Figure S2e). eGFP molarity was measured using a UV-Vis spectrophotometer and the published extinction coefficient at $488 \mathrm{~nm} .{ }^{50}$ Using these bulk solution calibrations, we can estimate the per-molar fluorescence of both eGFP and the bead solution at arbitrary gain, laser power, and pinhole settings.

Finally, we measured the average fluorescence intensity from single beads adsorbed to glass. Intensity was extracted from each image as the amplitude of a 2D Gaussian fit to each segmented localization. We measured single bead intensity over a range of instrument settings mirroring those used in the bulk calibration, and fit all the measurements to a similar arbitrary four-parameter "single" model of the form Counts $=A \times$ gain $+B \times$ laser $\times($ pinhole $-C) \times D^{\text {gain }}$ 
(Supplementary Figure S2c). This model allowed us to estimate the fluorescence of a single bead at arbitrary instrument settings.

The ratio of fluorescence from a single emitter to a bulk solution of emitters does not depend on the emitter type, Counts $\mathrm{single} /$ Counts $_{\text {bulk }}=k$, assuming that emitter concentration and imaging conditions are identical. ${ }^{51}$ Thus, we can solve for the fluorescence intensity of a single eGFP as eGFP single $=$ eGFP bulk $\times\left(\right.$ bead $_{\text {single }} /$ bead $_{\text {bulk }}$ ). The calibrations allow us to estimate single eGFP intensity at experimental gain, laser power, and pinhole settings, using the bulk per-molar fluorescence from both beads and eGFP calculated at the experimental settings from our 'bulk' model, and the fluorescence from single beads at the same settings using our 'single' model.

Single GFP quantification for occupancy estimation method 2: HiLo-TIRFM. In a complementary approach to method 1, we estimated the number of eGFPs within single foci using HiLoTIRFM. As HiLo-TIRFM is far more sensitive than confocal microscopy, we directly measured the fluorescence from single, purified eGFPs (CellBioLabs) adsorbed to plasmacleaned glass. We added eGFP to a solution of pH 7.4 PBS while providing simultaneous epiillumination $\left(0.3 \mathrm{~kW} / \mathrm{cm}^{2}\right) 488 \mathrm{~nm}$ laser until we began to see blinking events characteristic of single GFPs non-specifically adsorbing to the surface. ${ }^{44}$ The average eGFP intensity was calculated as the amplitude of a 2D Gaussian fit to each discernable spot. We then imaged ArrayG, without changing any instrument settings, and measured the distribution of singlemolecule intensities, again as the amplitude of a 2D Gaussian. We performed a separate eGFP intensity calibration each time each time we quantified ArrayG occupancy.

TIRF, dual color TIRF and HiLo-TIRFM imaging. Total internal reflection microscopy (TIRF or TIRFM), dual-color TIRFM, and HiLo-TIRFM, were performed on an Olympus CellTIRF system, with independent motor controlled TIRF angles for each fiber-coupled illumination laser, through a 1.49 NA 100x objective and 1.6x Optovar magnifier. Images were recorded on an Andor iXon Plus EMCCD camera at 20 frames per second, unless otherwise noted. Dual-color experiments were imaged through a Photometrics DualView 2 with simultaneous excitation of $488 \mathrm{~nm}$ and 561 or $639 \mathrm{~nm}$ lasers. All dichroics and filters were purchased from Semrock. All experiments were performed within a heated, $\mathrm{CO}_{2}$ controlled incubation chamber set to $37{ }^{\circ} \mathrm{C}$ and $5 \%$, respectively. Additional temperature control was provided by a collar-type objective heater, also set to $37^{\circ} \mathrm{C}$.

Single particle tracking. Single particle tracking (SPT) was performed in two steps, all using custom-written Python and $\mathrm{C}++$ code. First, detectable particles in each frame were identified by performing a Laplacian of Gaussian filter and then thresholding the filtered image based on intensity. A 2D Gaussian centered on each identified particle was then refined onto the raw image data using the Levenberg-Marquardt algorithm, with particle $x / y$ position, amplitude, background height, and in some instances standard deviation as free parameters. All particle detection parameters were validated by eye for each dataset, using a custom GUI. The single particle positions in each frame were then linked into single particle time trajectories using a nearest-neighbor type particle tracking heuristic, with a maximum per-frame jump distance parameter. The algorithm iteratively minimized the jump distance between frames, with no assumed momentum. All single particle trajectories were spot-validated by eye. For signal-to- 
noise ratio calculations, the signal was defined as the peak height of the Gaussian fit above background level, and noise was defined as the standard deviation of a $5 \times 5$ pixel window centered around the particle location after subtracting fitted Gaussian.

Fractional Brownian Motion: The raw $X Y, T$ data were directly fit to a three parameter fBM model (Mathematica version 11.1.1.0, FractionalBrownianMotionProcess $[\mu, \sigma, \quad h]$, ProcessEstimator $\rightarrow>$ "MaximumLikelihood"]). The first term $\mu$ captures constant 'drift', $\sigma$ captures the covariance $\left(=\sigma^{2}\left(\mathrm{~s}^{2 \mathrm{~h}}+\mathrm{t}^{2 \mathrm{~h}}-\mathrm{Abs}[\mathrm{t}-\mathrm{s}]^{2 \mathrm{~h}}\right) / 2\right)$, and $h$ is the Hurst parameter. Note that $h$ is simply $1 / 2$ of $\alpha$, the slope of LogLog transformed MSD. For ease of comparing to the existing literature, we report a rather than the Hurst parameter $h$.

$\begin{array}{lll} & \mathbf{0 . 5} \mathbf{~ H z} & \mathbf{2 0} \mathbf{~ H z} \\ \text { Number of cells: } & 8 & 11 \\ \text { Datapoints: } & 353605 & 103459 \\ \text { Number of trajectories: } & 1084 & 445 \\ \text { a Mean: } & 0.422115 & 0.434427 \\ \text { SD: } & 0.137685 & 0.163548 \\ 95 \% \mathrm{Cl} & \{0.41391,0.430321\} & \{0.41919,0.449664\}\end{array}$

Kinesin analysis. Starting with raw SPT data, we first removed stuck particles by filtering out tracks that moved less than $0.4 \mu \mathrm{m} / \mathrm{s}$ as measured by the distance between the first and last frame over the total track length. Freely diffusing particles and tracking failures (common at high particle density) were removed by filtering out tracks that turned too sharply, as measured by the maximum track curvature. Using the filtered subset, kinesin speed and run distance were measured by first smoothing each trajectory with a Savitzky-Golay filter to average away particle localization errors, making a one-dimensional distance axis. Within each trajectory, each localization was then assigned to the closest interpolated point along the smoothed distance axis, giving run distance as a function of time. Each distance versus time trajectory was fit to a simple linear function to measure run speed, and average speed was measured as the peak of the resultant speed distribution. In high time resolution data, free-diffusing and bound kinesins were distinguished by measuring a sliding window average centered around each time point, +/10 frames. Regions with a standard deviation larger than $30 \mathrm{~nm}$ were considered diffusing, and lower than $30 \mathrm{~nm}$ bound. Speed variation along each bound segment was measured as the first derivative of a Savitsky-Golay filter with a window size of 25 frames, and an order of 2. All analysis was performed using custom code in python.

Cell spreading assay. To achieve a high expression of the integrin $\beta 1$-ArrayG constructs, cells were induced with cumate at $5 x$ the working concentration diluted from a 10,000x water-soluble stock solution (SBI $300 \mathrm{mg} / \mathrm{mL}$ QA150A-1) for at least 3 days before assessing cell spreading. The $\mathrm{pKOaV}+\beta 1$-Array $\mathrm{G}_{16 x}+\mathrm{mGFP}$ cell line was cultured in the absence of tetracycline using Tet-free FBS, to prevent background induction of the Tet-on inducible control of mGFP. Four hours before performing the spreading assay, mGFP expression was induced with Doxycycline at $2 \mu \mathrm{g} / \mathrm{mL}$. This was done to promote efficient ER processing and subsequent trafficking of integrin to the membrane without GFP binding, and then subsequently assess functionality of the GFP-occupied array on integrin $\beta 1$ in the membrane. Glass bottomed imaging dishes (No. 1 imaging glass, Mattek) were incubated with $10 \mu \mathrm{g} / \mathrm{mL}$ fibronectin in PBS for 2 hours at $37^{\circ} \mathrm{C}$. 
pKOaV cell lines were then seeded on the fibronectin coated dishes and immediately placed on an confocal microscope (Zeiss LSM 700) with a live imaging incubation chamber equilibrated to $37^{\circ} \mathrm{C}$ and $6 \% \mathrm{CO}_{2}$. Following a 1-hour equilibration period, cells were imaged using the transmission photomultiplier tube (T-PMT) every 1 hour for 5 hours to obtain bright field images of an $8 \times 7$ tile (1145 $\mu \mathrm{m} \times 1000 \mu \mathrm{m}$ area). Image analysis took place in three steps: (1) stitching of raw image tiles using on-board Zeiss imaging software, (2) local contrast enhancement of stitched images followed by a Sobbel edge finding filter using ImageJ, (3) Segmentation of cells using custom software written in Python to find cell contours. Each cell contour checked by eye to match the raw images, and manually adjusted if needed. Calculation of mean spreading data used data from cells that remained within the image area and did not divide during the experiment. Data presented represents $N=50$ to 60 cells per condition (specific numbers given in figure) from two independent replicates. The sample sizes used here are similar to previous measurements. ${ }^{42}$

MSD analysis. Mean-square displacement (MSD) calculations were done using the $@$ @msdanalyzer class for MATLAB. ${ }^{52}$ When present, homogenous drift from the microscope stage was corrected using velocity correlations between individual tracks. After drift correction, the MSD was calculated directly from each trajectory.

Diffusion coefficient distribution. Integrins exhibit multiple modes of motion that are apparent in the distribution of diffusion coefficients. ${ }^{40}$ We estimated the diffusion coefficient for individual trajectories, rather than ensemble averaged MSD curves, so that we would not loose information about transitions among molecular behaviors. We calculated the distribution of single molecule diffusion coefficients by fitting Equation 2 over a specified time scale.

$$
<d^{2}(t)>=4 D t+\epsilon \quad \text { Eq. } 2
$$

Here, $\left\langle d^{2}>\right.$ is the MSD of an individual trajectory, $t$ is the displacement time scale, $D$ is the diffusion coefficient and $\varepsilon$ is an offset due to localization error. For all datasets, we used $t=0.33$ seconds to estimate $D$. Images of integrin $\beta 1-$ Array $_{16 x}$ were recorded at $20 \mathrm{~Hz}$, and integrin $\beta 1$ eGFP $_{2 x}$ images required $30 \mathrm{~Hz}$ recording to accurately capture its dynamics due to the faster diffusion rate. Because the integrin trajectories from ArrayG-labeled molecules sample long time-scales, it is likely that single molecules switch behaviors over a single trajectory, potentially masking the separate behaviors through averaging. Further, immobilized molecules are more easily tracked, and therefore can have much longer trajectory lengths than mobile molecules. To avoid these biases, we divided long trajectories into a series of non-overlapping 1 second segments, and estimated the diffusion coefficient as described above. Smooth distributions were generated using a normal kernel probability density estimation. To compare the relative diffusion rates of $\mathrm{GGP}_{2 \mathrm{x}}$ and ArrayG $_{16 \mathrm{x}}$, the log-scaled diffusion coefficient distribution was fit to a two-Gaussian mixture, giving a rate of $\mu \mathrm{m}^{2} / \mathrm{s}$ for $\mathrm{eGFP}_{2 \mathrm{x}}$ and $0.14 \mu \mathrm{m}^{2} / \mathrm{s}^{\text {ArrayG }} \mathrm{G}_{16 \mathrm{x}}$. Diffusion analysis inside vinculin-mCherry adhesions was performed as follows: (1) registration of GFP and mCherry channels using a rigid-body transformation of the mCherry channel onto the GFP channel from 405 illuminated sample images which produce the same features in each channel, (2) segmentation of time-averaged vinculin-mCherry using a Laplacian of Gaussians filter, and 
(3) assignment of trajectories that resided within contours of adhesions for $75 \%$ of their lifetime as 'inside' the adhesion.

Integrin trajectory segmentation using vbSPT. Integrin $\beta 1$-Array $\mathrm{G}_{16 \mathrm{x}}$ trajectories from either pKO- $\alpha \mathrm{V}$ or $-\alpha \mathrm{V} \beta 1$ cell lines were subjected to segmentation analysis using $\mathrm{vbSPT}^{43}$. Settings used were as follows: minimum track length $=20$ frames ( 1 second), initial diffusion coefficient range $=\left[10^{-4}, 1\right] \mu \mathrm{m}^{2} / \mathrm{s}$, initial dwell time range $=[0.5,25]$ seconds, runs $=16$, and default settings for prior distributions of diffusion coefficient, transition rates, and dwell times. Parameter estimations were determined from 100 bootstrap resamplings, besides state dwell times, which were taken as the arithmetic mean based on all trajectories in each state.

\section{Quantifying the dynamics of binder interaction with cognate arrays using FRAP to} determine affinity of each array/binder pair. U2OS tet-on cells were co-transfected with $\mathrm{H} 2 \mathrm{~B}$ ArrayGN or H2B-(Snurportin IBB) ${ }_{4 x}$-ArrayD and mwtGFP or DHFR-mGFP plasmids respectively. For ensemble FRAP arrays and binders were induced overnight with doxycycline and cumate. All FRAP analysis was carried out on cells with a typical interphase chromatin architecture, using a Zeiss 700 laser scanning confocal microscope enclosed in an incubation chamber set to $5 \% \mathrm{CO}_{2}$ and $37^{\circ} \mathrm{C}$. Cells were plated in Mattek glass bottomed dishes coated with fibronectin, and imaged using a $63 x$ oil-immersion objective. Bleaching was carried out using $488 \mathrm{~nm}$ laser operating at $100 \%$ laser power.

For measuring exchange of mwtGFP on ArrayGN using FRAP, two ROls per nucleus were generated $\left(\sim 8 \mu \mathrm{m}^{2}\right.$ per ROI). Background and bleach correction were done using ROls outside and inside the nucleus, respectively. Corrected intensity curves were fit to a single exponential decay, as free wtGFP contributes little to the fluorescent signal. For DHFR, we spot bleached a $\sim 2 \mu \mathrm{m}$ diameter circle to follow recovery. Average intensity as a function of time, I(t), was normalized to the average pre-bleach value. DHFR curves were fit with a double exponential function $\mathrm{I}(\mathrm{t})=\mathrm{A} 1+\mathrm{A} 2 \times \mathrm{EXP}\left(-\mathrm{k} 1^{*} \mathrm{t}\right)+\mathrm{A} 3 \times \mathrm{EXP}\left(-\mathrm{k} 2^{*} \mathrm{t}\right)(\mathrm{E} 1)$ to account for both the fast diffusive free binder-mGFP fraction and the less mobile fraction bound to the cognate scaffold. In case of the control cells expressing mGFP-NLS but no array, the intensity profile was fit with a single exponential function $\mathrm{I}(\mathrm{t})=\mathrm{A} 1+\mathrm{A} 2 \operatorname{xEXP}\left(-\mathrm{k} 1^{*} \mathrm{t}\right)$ (E2). The fitted parameters were averaged over $\sim 10$ repeats to compute means and standard deviations. Half maximum recovery was computed as $t_{1 / 2}=\log (0.5) /-k$.

Single molecule FRAP on H2B-ArrayGN. Exchange on arrays was also measured by imaging sparsely expressed H2B-ArrayGN, and performing FRAP on individual localizations. The initial spot intensity was measured by finding the maximum of the localization intensity for each spot in a z-stack of images. Subsequent bleaching was performed under constant illumination and recording $20 \mathrm{~Hz}$ images. Intensity measurements were taken at 0,30 , and 60 min postbleaching using the same procedure as initial spot measurements.

Data and code availability. All datasets and analysis software are available upon request.

\section{Acknowledgments}


This work was partially supported by the National Institutes of Health (NIH) National Institute Of General Medical Sciences (NIGMS)/National Cancer Institute (NCI) Grant GM77856, NCI Physical Sciences Oncology Center Grant U54CA143836, National Science Foundation Graduate Fellowship Program \#DGE-114747, and National Institute Of Biomedical Imaging And Bioengineering (NIBIB)/4D Nucleome Roadmap Initiative 1U01EB021237.

\section{Author information}

\section{Contributions}

R.P.G., J.M.F, W.D., Q.S., and J.T.L. designed research. R.P.G., J.M.F, W.D., and Q.S performed research. J.M.F., W.D., Q.S., and R.P.G. analyzed data. J.T.L., R.P.G., J.M.F, W.D. and Q.S wrote the paper.

\section{Competing financial interests}

None.

\section{Corresponding author}

Correspondence to: Jan Liphardt (jliphard@stanford.edu) 


\section{References}

1. Moffitt, J.R., Chemla, Y.R., Smith, S.B. \& Bustamante, C. Recent advances in optical tweezers. Annual review of biochemistry 77, 205-228 (2008).

2. Peterman, E.J., Sosa, H. \& Moerner, W.E. Single-molecule fluorescence spectroscopy and microscopy of biomolecular motors. Annu Rev Phys Chem 55, 79-96 (2004).

3. Lord, S.J., Lee, H.L. \& Moerner, W.E. Single-molecule spectroscopy and imaging of biomolecules in living cells. Analytical chemistry 82, 2192-2203 (2010).

4. Xia, T., Li, N. \& Fang, X. Single-molecule fluorescence imaging in living cells. Annu Rev Phys Chem 64, 459-480 (2013).

5. Cai, D., McEwen, D.P., Martens, J.R., Meyhofer, E. \& Verhey, K.J. Single molecule imaging reveals differences in microtubule track selection between Kinesin motors. PLOS biology 7, e1000216 (2009).

6. Buxbaum, A.R., Haimovich, G. \& Singer, R.H. In the right place at the right time: visualizing and understanding mRNA localization. Nature reviews. Molecular cell biology 16, 95-109 (2015).

7. Bertrand, E. et al. Localization of ASH1 mRNA particles in living yeast. Molecular cell 2, 437-445 (1998).

8. Robinett, C.C. et al. In vivo localization of DNA sequences and visualization of large-scale chromatin organization using lac operator/repressor recognition. The Journal of cell biology 135, 1685-1700 (1996).

9. Roukos, V., Burgess, R.C. \& Misteli, T. Generation of cell-based systems to visualize chromosome damage and translocations in living cells. Nature protocols 9, 2476-2492 (2014).

10. Chuang, C.H. et al. Long-range directional movement of an interphase chromosome site. Current biology : CB 16, 825-831 (2006).

11. Tanenbaum, M.E., Gilbert, L.A., Qi, L.S., Weissman, J.S. \& Vale, R.D. A protein-tagging system for signal amplification in gene expression and fluorescence imaging. Cell 159, 635-646 (2014).

12. Hui Liu et al. Stochastic Protein Labeling Enables Long-term Single Molecule Observation In Vivo. bioRxiv.org (2017).

13. Kamiyama, D. et al. Versatile protein tagging in cells with split fluorescent protein. Nat Commun 7, 11046 (2016).

14. Cabantous, S., Terwilliger, T.C. \& Waldo, G.S. Protein tagging and detection with engineered selfassembling fragments of green fluorescent protein. Nature biotechnology 23, 102-107 (2005).

15. Cabantous, S. \& Waldo, G.S. In vivo and in vitro protein solubility assays using split GFP. Nat Methods 3, 845-854 (2006).

16. Pinaud, F. \& Dahan, M. Targeting and imaging single biomolecules in living cells by complementation-activated light microscopy with split-fluorescent proteins. Proceedings of the National Academy of Sciences of the United States of America 108, E201-210 (2011).

17. Kerppola, T.K. Bimolecular fluorescence complementation (BiFC) analysis as a probe of protein interactions in living cells. Annu Rev Biophys 37, 465-487 (2008).

18. Leonetti, M.D., Sekine, S., Kamiyama, D., Weissman, J.S. \& Huang, B. A scalable strategy for high-throughput GFP tagging of endogenous human proteins. Proceedings of the National Academy of Sciences of the United States of America 113, E3501-3508 (2016).

19. De Meyer, T., Muyldermans, S. \& Depicker, A. Nanobody-based products as research and diagnostic tools. Trends in biotechnology 32, 263-270 (2014).

20. Muyldermans, S. Nanobodies: natural single-domain antibodies. Annual review of biochemistry 82, 775-797 (2013).

21. Kirchhofer, A. et al. Modulation of protein properties in living cells using nanobodies. Nat Struct Mol Biol 17, 133-U162 (2010).

22. Vale, R.D. The molecular motor toolbox for intracellular transport. Cell 112, 467-480 (2003).

23. Woehlke, G. et al. Microtubule interaction site of the kinesin motor. Cell 90, 207-216 (1997).

24. Zacharias, D.A., Violin, J.D., Newton, A.C. \& Tsien, R.Y. Partitioning of lipid-modified monomeric GFPs into membrane microdomains of live cells. Science 296, 913-916 (2002). 
25. Kim, J.H. et al. High cleavage efficiency of a $2 \mathrm{~A}$ peptide derived from porcine teschovirus-1 in human cell lines, zebrafish and mice. PLoS One 6, e18556 (2011).

26. Phair, R.D. \& Misteli, T. High mobility of proteins in the mammalian cell nucleus. Nature 404, 604609 (2000).

27. Lowe, A.R. et al. Selectivity mechanism of the nuclear pore complex characterized by single cargo tracking. Nature 467, 600-603 (2010).

28. Lieberman-Aiden, E. et al. Comprehensive Mapping of Long-Range Interactions Reveals Folding Principles of the Human Genome. Science 326, 289-293 (2009).

29. Wang, S. et al. Spatial organization of chromatin domains and compartments in single chromosomes. Science 353, 598-602 (2016).

30. Spichal, M. et al. Evidence for a dual role of actin in regulating chromosome organization and dynamics in yeast. J Cell Sci 129, 681-692 (2016).

31. Cabal, G.G. et al. SAGA interacting factors confine sub-diffusion of transcribed genes to the nuclear envelope. Nature 441, 770-773 (2006).

32. Hajjoul, $\mathrm{H}$. et al. High-throughput chromatin motion tracking in living yeast reveals the flexibility of the fiber throughout the genome. Genome Res 23, 1829-1838 (2013).

33. Weber, S.C., Spakowitz, A.J. \& Theriot, J.A. Nonthermal ATP-dependent fluctuations contribute to the in vivo motion of chromosomal loci. Proceedings of the National Academy of Sciences of the United States of America 109, 7338-7343 (2012).

34. Shinkai, S., Nozaki, T., Maeshima, K. \& Togashi, Y. Dynamic Nucleosome Movement Provides Structural Information of Topological Chromatin Domains in Living Human Cells. PLoS Comput Biol 12, e1005136 (2016).

35. Bronshtein, I. et al. Exploring chromatin organization mechanisms through its dynamic properties. Nucleus 7, 27-33 (2016).

36. Oyen, D., Wechselberger, R., Srinivasan, V., Steyaert, J. \& Barlow, J.N. Mechanistic analysis of allosteric and non-allosteric effects arising from nanobody binding to two epitopes of the dihydrofolate reductase of Escherichia coli. Biochimica et biophysica acta 1834, 2147-2157 (2013).

37. Katrukha, E.A. et al. Probing cytoskeletal modulation of passive and active intracellular dynamics using nanobody-functionalized quantum dots. 8, 14772 (2017).

38. Perillo, E.P. et al. Deep and high-resolution three-dimensional tracking of single particles using nonlinear and multiplexed illumination. 6, 7874 (2015).

39. Ibach, J. et al. Single Particle Tracking Reveals that EGFR Signaling Activity Is Amplified in Clathrin-Coated Pits. PLOS ONE 10, e0143162 (2015).

40. Rossier, $O$. et al. Integrins beta1 and beta3 exhibit distinct dynamic nanoscale organizations inside focal adhesions. Nature cell biology 14, 1057-1067 (2012).

41. Shibata, A.C. et al. Archipelago architecture of the focal adhesion: membrane molecules freely enter and exit from the focal adhesion zone. Cytoskeleton (Hoboken) 69, 380-392 (2012).

42. Schiller, H.B. et al. beta1- and alphav-class integrins cooperate to regulate myosin II during rigidity sensing of fibronectin-based microenvironments. Nature cell biology 15, 625-636 (2013).

43. Persson, F., Linden, M., Unoson, C. \& Elf, J. Extracting intracellular diffusive states and transition rates from single-molecule tracking data. Nat Methods 10, 265-269 (2013).

44. Leduc, $\mathrm{C}$. et al. A highly specific gold nanoprobe for live-cell single-molecule imaging. Nano letters 13, 1489-1494 (2013).

45. $\mathrm{Li}$, J. et al. Conformational equilibria and intrinsic affinities define integrin activation. The EMBO Journal 36, 629-645 (2017).

46. Kong, F., García, A.J., Mould, A.P., Humphries, M.J. \& Zhu, C. Demonstration of catch bonds between an integrin and its ligand. The Journal of cell biology 185, 1275-1284 (2009).

47. Roca-Cusachs, P., Gauthier, N.C., Del Rio, A. \& Sheetz, M.P. Clustering of alpha(5)beta(1) integrins determines adhesion strength whereas alpha(v)beta(3) and talin enable mechanotransduction. Proceedings of the National Academy of Sciences of the United States of America 106, 16245-16250 (2009).

48. Schvartzman, M. et al. Nanolithographic control of the spatial organization of cellular adhesion receptors at the single-molecule level. Nano letters 11, 1306-1312 (2011).

49. Viswanathan, S. et al. High-performance probes for light and electron microscopy. Nat Methods 12, 568-576 (2015). 
bioRxiv preprint doi: https://doi.org/10.1101/159004; this version posted July 3, 2017. The copyright holder for this preprint (which was not certified by peer review) is the author/funder. All rights reserved. No reuse allowed without permission.

50. McRae, S.R., Brown, C.L. \& Bushell, G.R. Rapid purification of EGFP, EYFP, and ECFP with high yield and purity. Protein expression and purification 41, 121-127 (2005).

51. Graham, J.S., Johnson, R.C. \& Marko, J.F. Counting proteins bound to a single DNA molecule. Biochemical and biophysical research communications 415, 131-134 (2011).

52. Tarantino, N. et al. TNF and IL-1 exhibit distinct ubiquitin requirements for inducing NEMO-IKK supramolecular structures. The Journal of cell biology 204, 231-245 (2014). 


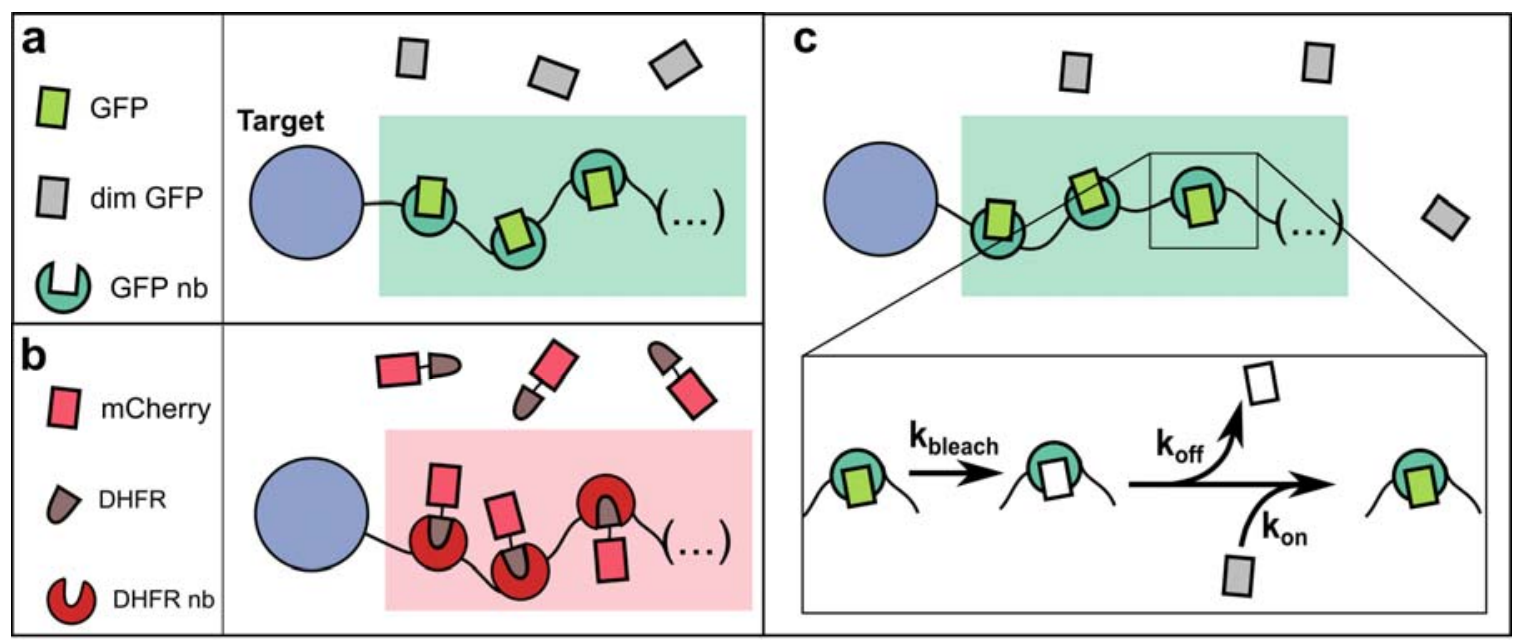

d
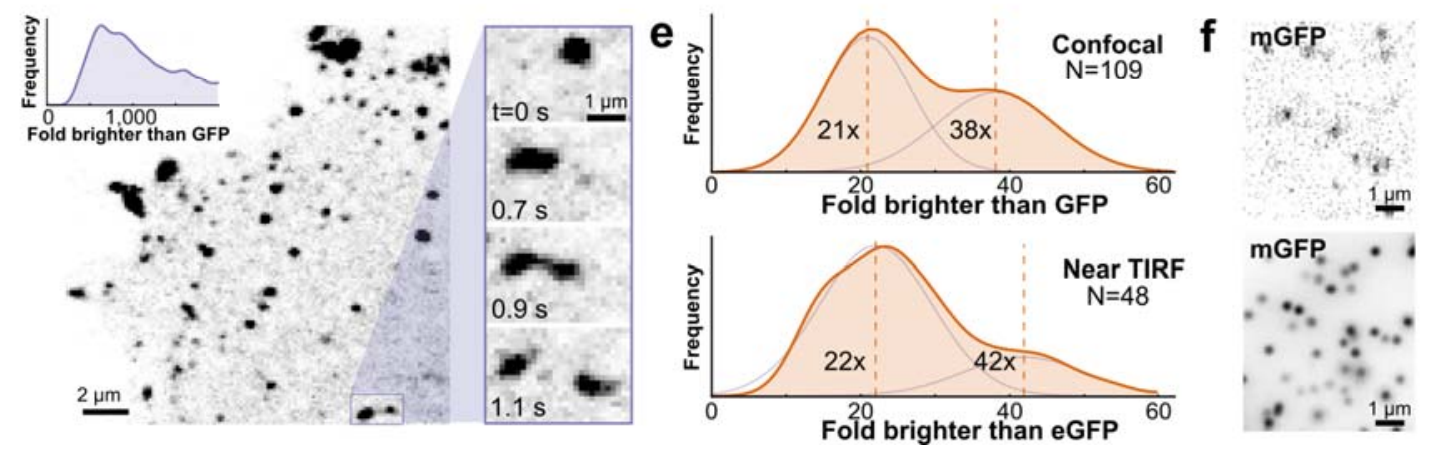

Figure 1. A dynamic recruitment based approach for prolonged imaging and abolishing aggregation propensity. (a) A schematic representation of dynamic recruitment on the the fluorogenic GFP-nanobody array. (b) A schematic representation of dynamic recruitment of DHFR-mCherry on DHFR-nanobody array. (c) Depiction of fluorescence sustenance on an array through exchange of bound photo-bleached fluorophores with dim unbound fluorophores which subsequently brighten upon binding (d) Representative laser-scanning confocal image of a cell co-expressing kinesin-1(KIF560)- (GBP1 $24 x$ GFP-nanobody array) along with eGFP as the binder. Right inset: Zoom-in showing droplet-like temporal dynamics of kinesin aggregates. Top left inset: Distribution of intensities of spots in terms of fold brighter than eGFP, showing extensive aggregation. The x-axis has been truncated at 2,000 eGFPs to emphasize the large size of even the smallest aggregates. (e-f) The use of monomeric A206K GFP (mGFP) abolishes aggregation. KIF560-GBP1 $1_{24 x}$ intensity distributions represented as fold-brighter than eGFP, show peaks corresponding to KIF560-GBP1 $1_{24 x}$ monomer and dimer for confocal (top) and HiLo-TIRF (bottom) (e), with representative images (f). Intensity distributions were fit to a twoGaussian mixture model to estimate the local maxima for the monomeric and dimeric peaks. Vertical lines in intensity distributions represent the mean value. 

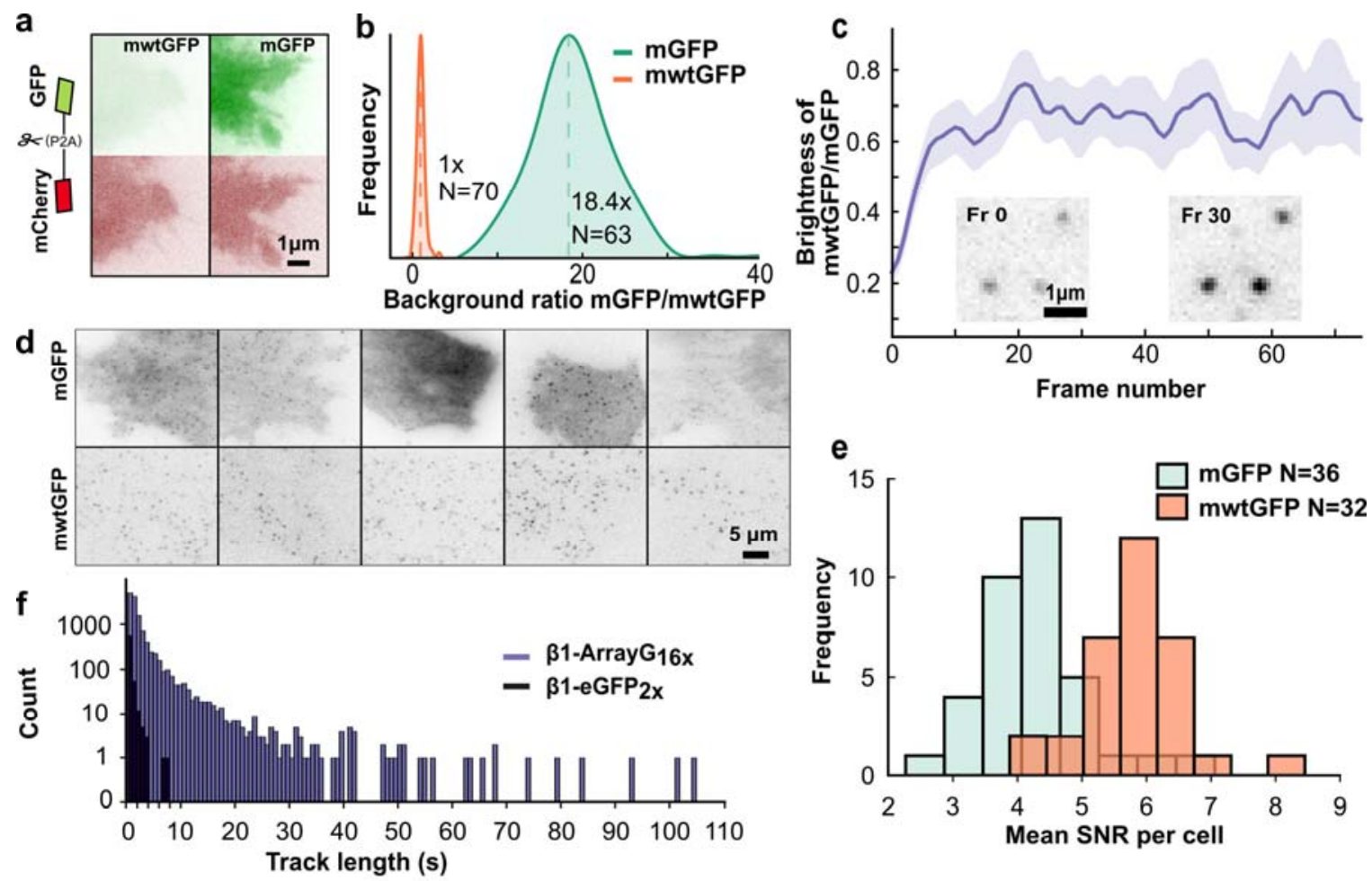

Figure 2. Fluorescence enhancement of mwtGFP upon binding GBP1 array suppresses background fluorescence. (a) TIRF images of HeLa cell lines stably expressing self-cleavable constructs for ratiometric normalization of GFP fluorescence: mCherry-p2a-mwtGFP (left) or mCherry-p2a-mGFP (right). (b) Distribution of mGFP intensity ( $\mathrm{N}=63$ cells) normalized by mean mwtGFP intensity ( $\mathrm{N}=70$ cells) measured using TIRF. Vertical dashed lines represent mean value. (c) Time dependence of $\mathrm{GBP}_{24 x}$ array bound mwtGFP brightness relative to mGFP. Inset: TIRF images of mwtGFP+GBP1 $1_{24 x}$ spots at frame 0 and frame 30 (at $20 \mathrm{~Hz}$ image frequency), showing significant brightening. (d) TIRF images of U2OS cells stably expressing integrin $\beta 1$-Array $G_{16 x}$ and either mGFP (top) or mwtGFP (bottom) binder. (e) Histogram of the mean array signal-to-noise per cell for integrin $\beta 1$-Array $G_{16 x}$ co-expressing either $m G F P$ or mwtGFP. (f) Trajectory length distribution for $\beta 1$-eGFP $2 x$ (659 trajectories from 4 cells) and $\beta 1$ Array $_{16 x}(13,423$ trajectories from 4 cells). Minimum trajectory length is 10 frames. 

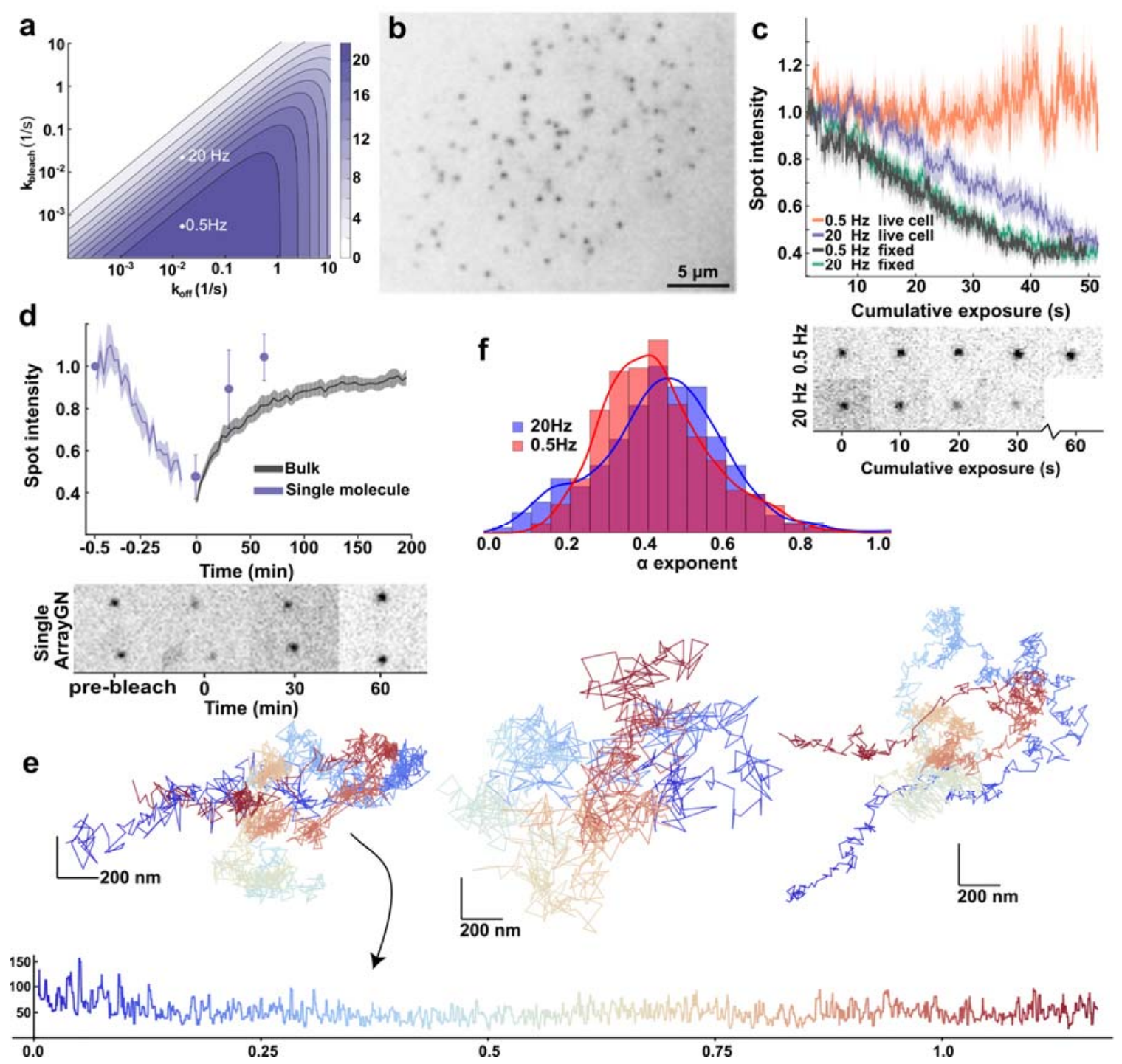

Figure 3. Hour-duration tracking of single histones in the nucleus using ArrayGN. (a) Theoretical prediction of array occupancy by unbleached fluorophores as a function of bleach rate, $k_{\text {bleach, }}$ and unbinding rate, $k_{\text {off }}$ (plotted using $k_{\text {on }}=10$ ). The occupancy estimations for $20 \mathrm{~Hz}$ and $0.5 \mathrm{~Hz}$ imaging are shown as white crosses. (b) HiLo-TIRF image of a nucleus with integrated H2B-ArrayGN ${ }_{24 x}$. (c) Top: Normalized spot intensity for tracked single-molecules as a function of cumulative laser exposure for $20 \mathrm{~Hz}$ or $0.5 \mathrm{~Hz}$ (50 ms exposure/frame) in fixed and live cells. (0.5 Hz live: $N=20 ; 0.5 \mathrm{~Hz}$ fixed: $N=24,20 \mathrm{~Hz}$ live: $N=19 ; 20 \mathrm{~Hz}$ fixed: $N=23$ ). Solid lines are mean intensity, shading is s.e.m. Bottom: raw images of single spots of H2BArrayGN ${ }_{24 x}$ for $20 \mathrm{~Hz}$ and $0.5 \mathrm{~Hz}$ in live cells. (d) Top: FRAP for single H2B-Array $\mathrm{GN}_{24 x}$ tracked over time ( $N=3$ traces) and bulk FRAP from nuclear regions in cells overexpressing H2BArrayG $_{N}$ and wtmGFP ( $N=5$ cells, 2 ROIs per cell). Solid line is mean intensity, shading and error bars are s.e.m. Bottom: raw image of single H2B-ArrayGNN ${ }_{24 x}$ FRAP. (e) Three examples of single histone trajectories, color coded by time. Bottom: A five-point median-filtered plot of a single histone's displacements over time. (f) Distribution of a values for H2B-ArrayGNN ${ }_{24 x}$ imaged at $0.5 \mathrm{~Hz}$ and $20 \mathrm{~Hz}$. 

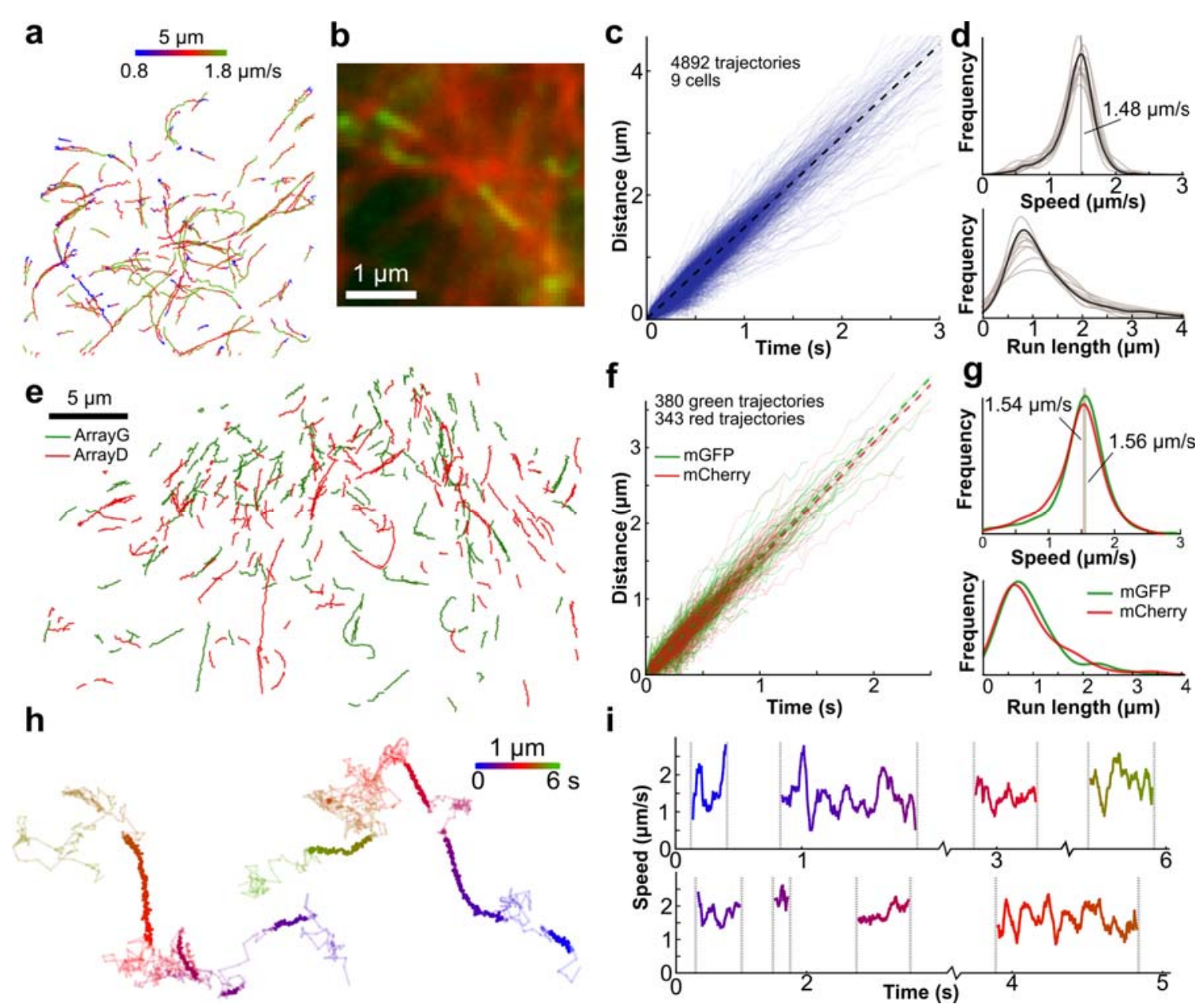

Figure 4. Kinesin-ArrayG trajectories demonstrates proper functionality and reveals large speed fluctuations. (a) Trajectories of single KIF560-ArrayG ${ }_{24 x}$ motors labeled with mwtGFP colored by speed. (b) Time projection of KIF560-Array $\mathrm{G}_{24 x}$ at $80 \mathrm{~Hz}$ on labeled tubulin. (c) Distance versus time trace for 4892 individual trajectories from 9 cells. Dashed line represents the mean speed. (d) Distribution of trajectory speeds (top) and run lengths (bottom) from c. Individual cell distributions are shown in transparent, with the mean as solid. Vertical dashed line indicates the mean $(1.5 \mu \mathrm{m} / \mathrm{s})$. (e) Trajectories of kinesin in cells co-expressing KIF560ArrayG $_{24 x}+$ mwtGFP (green) and KIF560-ArrayD ${ }_{24 x}+$ DHFR-mCherry (red). (f) Distance versus time trace of trajectories shown in $\mathbf{e}$. (g) Distribution of speed and run length from $\mathbf{f}$. (h) A high frame-rate trajectory of KIF560-ArrayG $(180 \mathrm{~Hz})$ colored by time. Linearly persistent trajectory segments are colored bold. (i) Instantaneous speed of the linearly persistent segments from the trajectories shown in $\mathbf{h}$. 

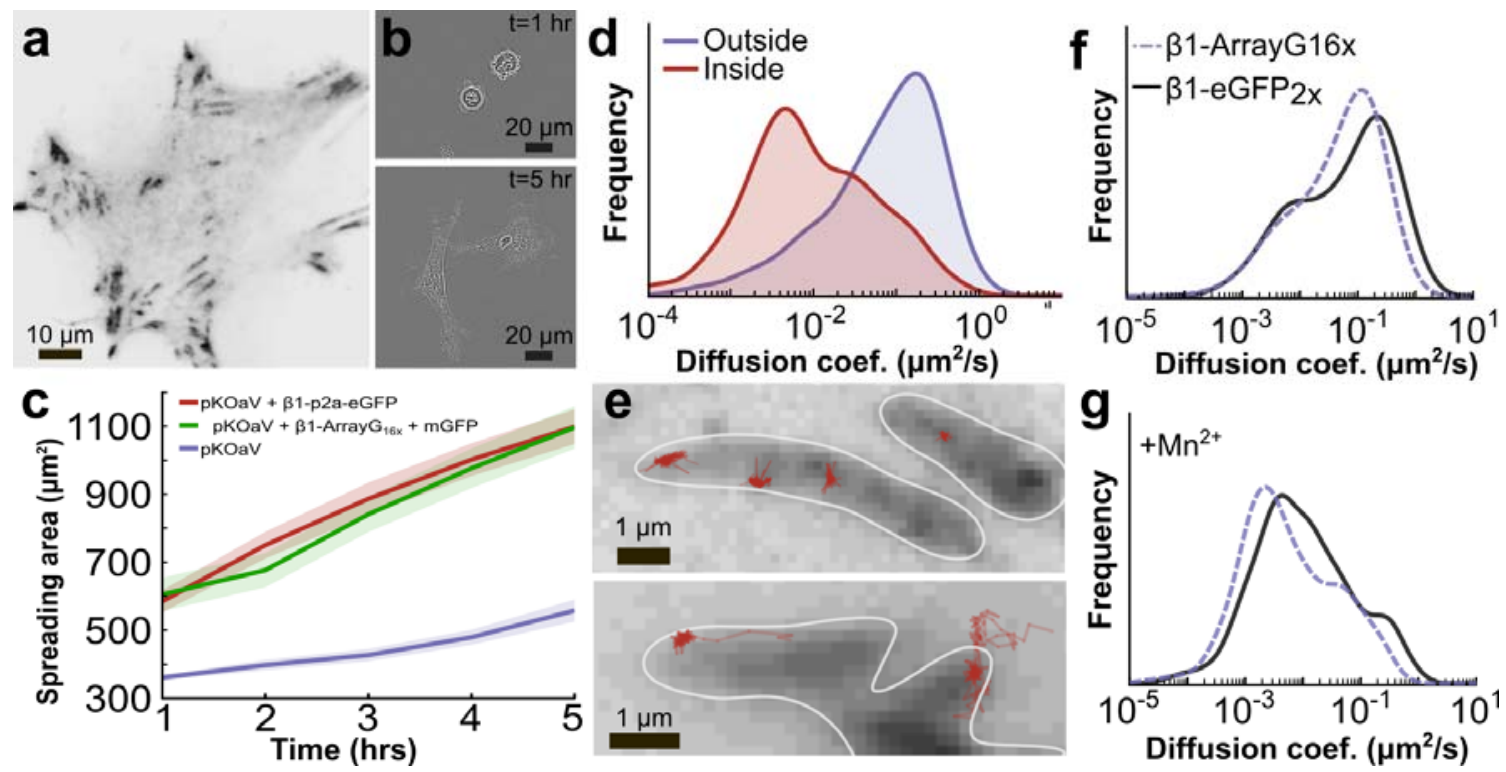

Figure 5. Evaluation of integrin $\beta 1-$ Array $_{16 x}$ functionality. (a) Representative TIRF image of pKOaV MEF cells after induction of integrin $\beta 1$-Array $G_{16 x}$ and mGFP. (b) Brightfield images of pKOaV MEFs overexpressing $\beta 1$-Array $G_{16 x}+$ mGFP at 1 and 5 hours post seeding on fibronectin. (c) Mean spreading area versus time for $p K O \alpha V$ (53 cells), $p K O \alpha V+\beta 1-(p 2 a)-e G F P$ (59 cells), and $p K O \alpha V+\beta 1-A r r a y G_{16 x}+m G F P$ (57 cells). Shaded area represents s.e.m. (d) Diffusion coefficient distribution of integrin $\beta 1-$ Array $_{16 x}$ inside and outside focal adhesions. $N=5$ cells, 1852 trajectory segments. (e) Examples of immobilized trajectories inside focal adhesions (top) and trajectories transitioning between diffusive and immobilized states at focal adhesion boundaries (bottom). Focal adhesion boundaries highlighted in white. (f) Distribution of diffusion coefficients of $\beta 1$-eGFP ${ }_{2 x}(N=4$ cells, 659 trajectories $)$ and $\beta 1-$ ArrayG $_{16 x}(N=9$ cells, 2860 trajectory segments) in untreated cells. (g) Distribution of diffusion coefficients after $2 \mathrm{mM} \mathrm{Mn}^{2+}$ treatment ( $\beta 1$-eGFP $2 x: N=3$ cells, 350 trajectories, $\beta 1-$ Array $_{16 x}: N=8$ cells, 1388 trajectory segments). 


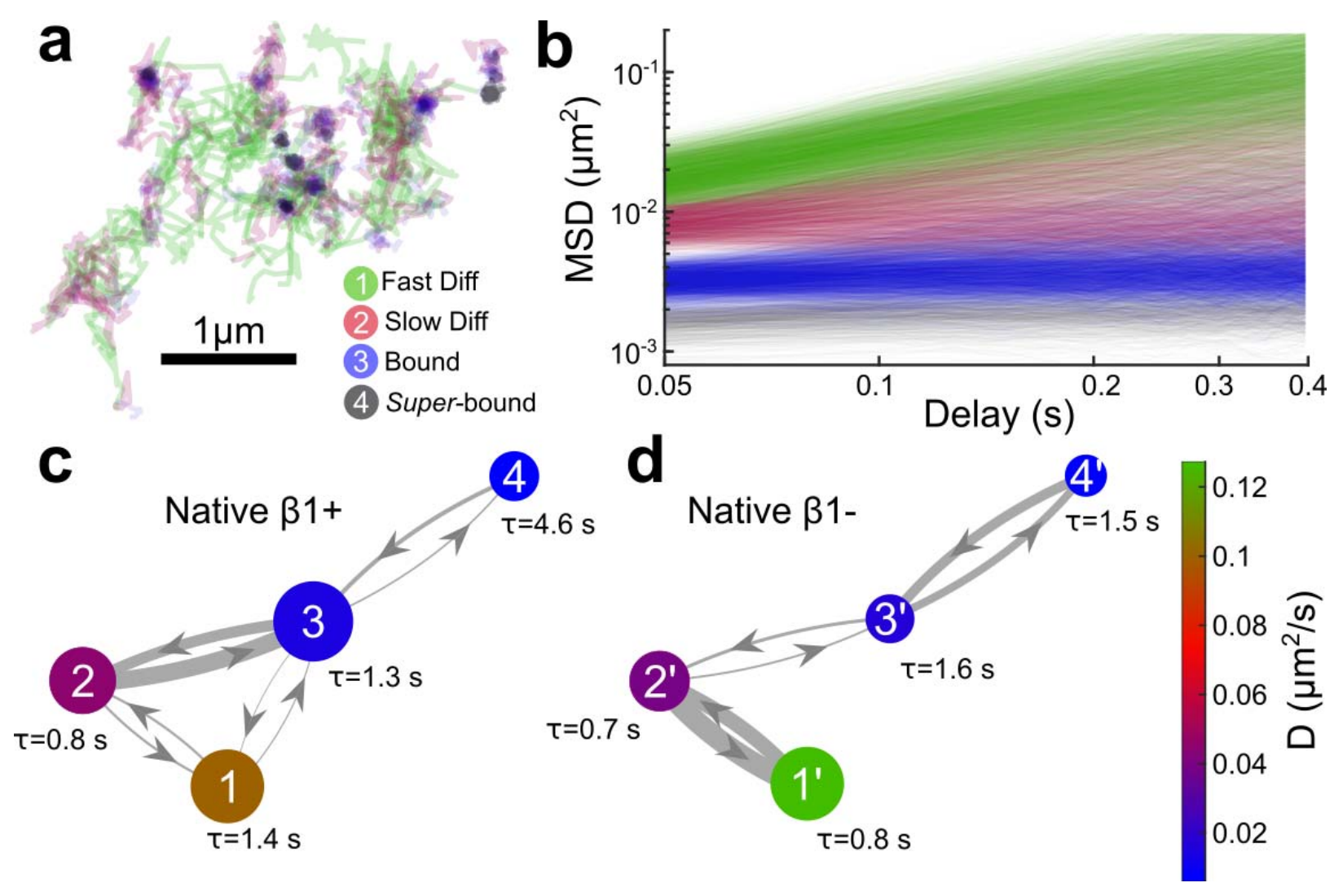

Figure 6. Hidden Markov trajectory segmentation using vbSPT. (a) Segmented trajectories color coded by state. (b) Overlayed MSD of individual trajectory segments for each state. (c, d) Transition state diagrams of $\beta 1-$ Array $_{16 x}$ in cells expressing native $\beta 1$ (pKOaV $\beta 1, N=9,669$ trajectories) (c) and in $\beta 1$ null cells (pKOaV, $N=6,138$ trajectories) (d). The area of each state corresponds to the relative occupation (normalized to state 1), the colors correspond to the diffusion coefficient (see colorbar), the width of connecting lines corresponds to the relative transition rate, and the mean state lifetime is written as $\mathbf{T}$ in seconds. Transition rates less than 0.01 per second are ignored. 